\title{
Análisis de la simetría del tamaño dentario mesiodistal de la misma muestra en dentición mixta y permanente. Estudio longitudinal
}

\author{
Austro Martínez M.D.*, Ostos Garrido M.J.***, \\ García Ballesta C.***, Pérez Lajarín L.****
}

\section{RESUMEN}

En el presente trabajo se estudian los diámetros mesiodistales de los dientes temporales y permanentes de la misma muestra y se analiza la existencia de simetría entre dientes homólogos. Dicho estudio fue realizado inicialmente sobre una muestra de 267 niños con dentición mixta, 90 (34\%) niñas y 177 (66\%) niños, de Andalucía Oriental, con edades comprendidas entre 8 y 10 años, transcurridos cuatro años volvimos a analizar a los mismos niños con dentición permanente, y sólo obtuvimos una muestra de 171, de los cuales 69 (40\%) eran niñas y el $102(60 \%)$ niños, con una edad media de 12 años y un rango de 11 a 13 años, el $21 \%$ de la muestra tenía 11 años, el 46,29\% presentaba una edad de 12 y el 32\% de 13 años, utilizándose como criterios de selección, que no tuvieran alteraciones morfológicas, pérdida dentarias, apiñamientos importantes, entre otros factores. El tamaño dentario se midió, como la máxima distancia entre los puntos de contacto mesial-distal de la corona, utilizándose un calibre de punta fina, con una precisión de $0,1 \mathrm{~mm}$. Todas las mediciones fueron realizadas por el mismo observador, directamente en boca, utilizando luz natural y espejos desechables.

El análisis estadístico incluyó pruebas como la "t" de Student, con un nivel de significación estadística de p< 0,05. Los principales resultados incluyen la existencia de simetría entre dientes homólogos, tanto en dentición temporal como en dentición permanente.

Palabras claves: Tamaño mesiodistal de los dientes, simetría.

\section{ABSTRACT}

In the present work is analysed the mesiodistal diameters of the temporal and permanent teeth of the same sample to calculate the symmetry.

The first sample was 267 children, 90 girls (34\%) and 177 (66\%) boys from Oriental Andalusia, aged between 8 and 10 years old. After four years, we analysed the same children with permanent teething and we obtained just a sample of 171, 69 girls (40\%) and $102(60 \%)$ boys, with an average age of 12 years and a range from 11 to 13 years, using as criteria selection not having morphological alteration, teeth loss, important congestions, between other elements.

The teething dimension was measured as the maximum distance between the mesiodistal contact points of the crown, using a sharp-end gauge, with a $0,1 \mathrm{~mm}$ precision. All the measurements were made by the same viewer, directly to the mouth. For the statistic analysis was used the $t$ Student test, with a level of statistic signification of $p<0,05$. 
The main results include the symmetry of the mesiodistal size both in a mixed dentition and a permanent dentition, of the same sample.

Key words: Mesiodistal size teeth,symmetry.

Aceptado para publicación: noviembre 2003.

* Profesor Asociado. Facultad de Odontología. Universidad de Murcia.

** Profesor Titular. Facultad de Odontología. Universidad de Granada.

**** Profesor Titular. Facultad de Odontología. Universidad de Murcia.

Austro Martínez M.D., Ostos Garrido M.J., García Ballesta C., Pérez Lajarín L. Análisis de la simetría del tamaño dentario mesiodistal de la misma muestra en dentición mixta y permanente. Estudio longitudinal. $A v$. Odontoestomatol 2004; 20-6: 305-313.

\section{INTRODUCCIÓN}

El conocimiento del tamaño de los dientes temporales y permanentes es muy útil tanto en antropología como en odontología.

Numerosos autores han estudiado los dientes permanentes, tanto desde el punto de vista morfológico como métrico $(1,2,3,4,5,6)$. Sin embargo son menos los trabajos de investigación publicados sobres dichos aspectos en la dentición temporal $(7,8,9,10)$. En la práctica ortodóncica y odontopediátrica es fundamental conocer la relación entre el tamaño de los molares temporales y sus sucesores permanentes. La forma y el tamaño de las coronas se establecen nítidamente en edades tempranas y sus cambios debidos al paso del tiempo son escasos, únicamente se producen por el desgaste a causa del uso, por enfermedades o por traumatismos y se ven poco influidas por la acción ambiental durante el período formativo (11).

El tamaño de los dientes viene determinado principalmente por la herencia $(12,2,4)$.

De todas las mediciones la que recibió mayor atención en la literatura ortodóncica, fue el diámetro mesiodistal, ya que es la dimensión que más relacionada está directamente con las maloclusiones.

Un aspecto a tener en cuenta es la simetría bilateral del diámetro mesiodistal. En los estudios realizados al respecto no se han encontrado patrones típicos de asimetría, que parece ocurrir al azar y, en general, su magnitud es poco importante, del orden de 0,1 mm o menor. $(13,14)$.

\section{OBJETIVOS}

1. Obtener los tamaños mesiodistales de la misma población con dentición mixta y dentición permanente.

2. Observar si existen diferencias significativas entre las mediciones realizadas en boca y en los modelos.

3. Examinar si existe simetría entre dientes homólogos en niños y niñas tanto en dentición temporal, como en dentición permanentes.

\section{MATERIAL Y MÉTODO}

La muestra estudiada constó inicialmente con 269 niños con dentición mixta, de los cuales 90 (34\%) eran niñas y el 177 (60\%) niños, con una edad media de 9 años, y un rango de 8 a 10 años, dicha muestra fue obtenida de escolares de Andalucía Oriental, concretamente de Jaén y provincia, a los cuales se les realizó la medición del tamaño mesiodistal de sus dientes. Transcurridos cuatro años, volvimos a analizar a los mismos niños, obteniendo sólo un tamaño muestral de 171, debido a la dificultad de la recogida de los datos, ya que los niños con 12 años pasan a estudiar de los colegios a diferentes Institutos, lo cual nos dificultó muchísimo la obtención de la misma. Por tanto, la muestra estudiada finalmente fue de 171 niños con dentición permanente, de los cuales 69 eran niñas (40\%) y 102 niños (60\%), con una edad media de 12 años y un rango de 11 a 13 años, el $21 \%$ tenían 11 años, el $46 \% 12$ y el 33\% 13 años.

En cuanto a los criterios de selección utilizados destacamos entre otros: 
$1^{\circ}$. Ausencia de anomalías en número, forma, o tamaño dentario.

$2^{\circ}$. Ausencia de pérdidas de sustancia dentaria debido a atriciones, caries, coronas, fracturas o elevado grado apiñamiento.

$3^{\circ}$. Sin antecedentes de tratamiento ortodóncico.

$4^{\circ}$. Erupción completa de los dientes.

Todas las mediciones fueron realizadas con un calibre de la marca Leone, con una precisión de $0,1 \mathrm{~mm}$, llevadas a cabo en todos los casos por el mismo observador, empleando luz natural y espejos desechables. Dichas mediciones fueron efectuadas directamente en boca. Para hallar si existían discrepancias entre las mediciones sobre modelos y las mediciones en boca, se utilizó una muestra de 50 pacientes de la Facultad de Odontología de Granada, a quienes se les realizó la medición de sus dientes en boca y en sus modelos. No encontrándose diferencias significativas, por lo que se dedujo que el método utilizado era válido.

El tamaño mesiodistal de acuerdo con Moorrees, se midió como la máxima distancia entre los puntos de contacto mesial y distal, poniendo el eje del calibre paralelo a las superficies oclusales o incisales.

Los datos de los tamaños mesiodistales han sido procesados empleando el paquete estadístico SPSS 9.0 para Windows. Después de una depuración de los mismos buscando los valores que estaban fuera del rango y las incoherencias entre variables, se obtuvo una distribución de frecuencias resumiendo los valores de los distintos grupos, en cuanto a variables cuantitativas, mediante media y desviación estándar. Las comparaciones de dos medias de tamaños mesiodistales en muestra apareadas, como el estudio inicial que se realizó para comparar las mismas mediciones, en los mismos pacientes en modelos y en boca, se realizaron con el test de la " $t$ " de Student.

\section{RESULTADOS}

No se encontraron diferencias significativas entre las mediciones realizadas en boca y en los modelos. (Tabla 1, Fig 1).

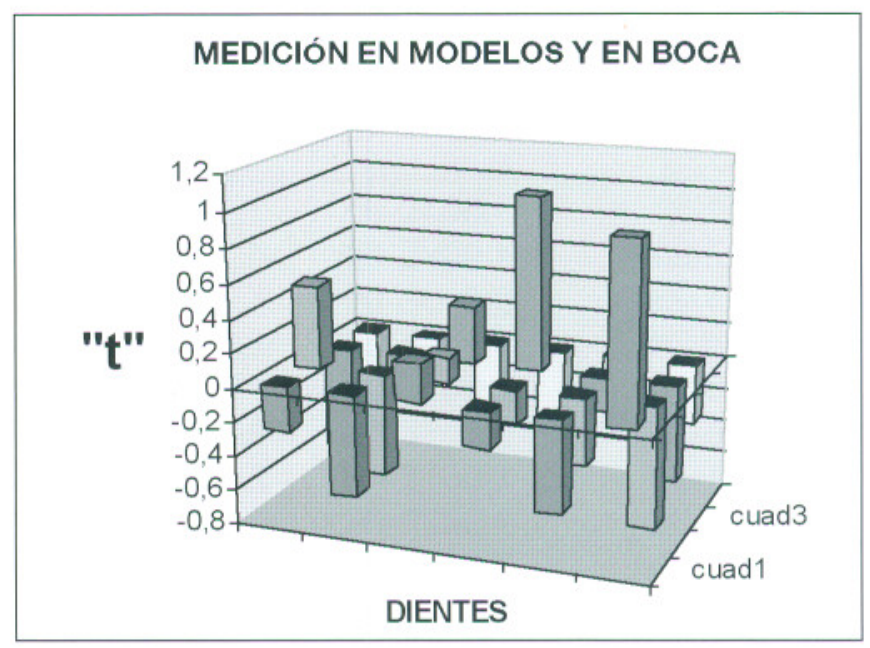

\begin{tabular}{|c|c|c|c|}
\hline \multicolumn{4}{|c|}{ TABLA 1.- MEDICIÓN DEL TAMAÑO } \\
MESIODISTAL EN BOCA Y EN LOS \\
MODELOS «t» DE STUDENT \\
\hline DIENTES & "t» & DIENTES & "t " \\
\hline 11 & -0.26 & 41 & -0.59 \\
21 & 0.49 & 31 & -0.61 \\
12 & -0.59 & 42 & -0.17 \\
22 & -0.60 & 32 & -0.16 \\
53 & 0.23 & 83 & -0.44 \\
63 & 0.15 & 73 & 0.34 \\
54 & -0.21 & 84 & -0.44 \\
64 & -0.20 & 74 & 1.01 \\
55 & -0.53 & 85 & -0.23 \\
65 & -0.39 & 75 & -0.20 \\
16 & 1.10 & 46 & -0.35 \\
26 & -0.70 & 36 & -0.57 \\
\hline
\end{tabular}

Al comparar el tamaño mesiodistal de los dientes permanentes en el lado derecho e izquierdo, observamos que no existen diferencias significativas entre las medias de las medidas mesiodistales para cada diente entre el lado derecho e izquierdo excepto para el 15 y 25 aunque esta diferencia no es muy significativa y para el 13 y 23 (Tabla 2, Tabla 3 ).

Al realizar el test de la " $t$ " de Student entre muestras relacionadas, obtenemos que no existen diferencias entre dientes homólogos excepto para 15 y 25 en niños, y 13 y 23 en niñas.

Hemos obtenido unos niveles de correlación altísimos, al analizar la correlación entre dientes homólogos en niños, observamos una correlación de 0,99 para casi 


\begin{tabular}{|l|c|c|c|c|c|c|}
\hline \multicolumn{6}{|c|}{ TABLA 2.- COMPARACIÓN DEL TAMAÑO M-D ENTRE DIENTES HOMÓLOGOS EN NIÑOS } \\
\hline PARES & MEDIA & DES. TIP. & $\begin{array}{c}\text { ERROR } \\
\text { TIP. MEDIA }\end{array}$ & $\begin{array}{c}\text { INTERVALO } \\
\text { INFERIOR }\end{array}$ & $\begin{array}{c}\text { INTERVALO } \\
\text { SUIPERIOR }\end{array}$ & " " \\
\hline P 11-21 & $-9.803 E-05$ & $7.379 E-03$ & $7.306 \mathrm{E}-04$ & $-1.547 \mathrm{E}-03$ & $1.351 \mathrm{E}-03$ & -0.134 \\
P 12-22 & $-4.902 \mathrm{E}-04$ & $4.700 \mathrm{E}-04$ & $4.777 \mathrm{E}-04$ & $-1.422 \mathrm{E}-03$ & $4.421 \mathrm{E}-04$ & -1.043 \\
P 13-23 & $-9.803 \mathrm{E}-05$ & $4.101 \mathrm{E}-03$ & $4.061 \mathrm{E}-04$ & $-9.036 \mathrm{E}-04$ & $7.076 \mathrm{E}-04$ & -0.241 \\
P 14-24 & $-3.921 \mathrm{E}-04$ & $4.203 \mathrm{E}-03$ & $4.162 \mathrm{E}-04$ & $-1.217 \mathrm{E}-03$ & $4.334 \mathrm{E}-04$ & -0.942 \\
P 15-25 & $2.647 \mathrm{E}-03$ & $1.226 \mathrm{E}-02$ & $1.214 \mathrm{E}-03$ & $2.386 \mathrm{E}-04$ & $5.055 \mathrm{E}-03$ & 2.180 \\
P 16-26 & $-9.803 \mathrm{E}-04$ & $9.901 \mathrm{E}-03$ & $9.804 \mathrm{E}-04$ & $-2.925 \mathrm{E}-03$ & $9.644 \mathrm{E}-04$ & -1.000 \\
P 41-31 & $1.961 \mathrm{E}-04$ & $2.429 \mathrm{E}-03$ & $2.405 \mathrm{E}-04$ & $-2.810 \mathrm{E}-04$ & $6.732 \mathrm{E}-04$ & 0.815 \\
P 42-32 & $3.922 \mathrm{E}-04$ & $3.122 \mathrm{E}-03$ & $3.091 \mathrm{E}-04$ & $-2.210 \mathrm{E}-04$ & $1.005 \mathrm{E}-03$ & 1.269 \\
P 43-33 & $2.941 \mathrm{E}-04$ & $2.616 \mathrm{E}-03$ & $2.590 \mathrm{E}-04$ & $-2.197 \mathrm{E}-04$ & $8.079 \mathrm{E}-04$ & 1.136 \\
P 44-34 & $-1.960 \mathrm{E}-04$ & $4.446 \mathrm{E}-03$ & $4.402 \mathrm{E}-04$ & $-1.069 \mathrm{E}-03$ & $6.771 \mathrm{E}-04$ & -0.445 \\
P 45-35 & $5.882 \mathrm{E}-04$ & $4.839 \mathrm{E}-03$ & $4.791 \mathrm{E}-04$ & $-3.621 \mathrm{E}-04$ & $1.539 \mathrm{E}-03$ & 1.228 \\
P 46-36 & $8.333 \mathrm{E}-03$ & $5.588 \mathrm{E}-02$ & $5.533 \mathrm{E}-03$ & $-2.643 \mathrm{E}-03$ & $1.931 \mathrm{E}-02$ & 1.506 \\
\hline
\end{tabular}

\begin{tabular}{|c|c|c|c|}
\hline \multicolumn{4}{|c|}{ TABLA 3.- COMPARACIÓN ENTRE } \\
\hline PARES & MEDIA & DES. TIP. & TIP \\
\hline P 11-21 & $-8.695 E-04$ & $6.121 E-03$ & 7.36 \\
P 12-22 & $-1.014 E-03$ & $6.891 E-03$ & 8.29 \\
P 13-23 & $-1.449 E-03$ & $3.939 E-03$ & 4.7 \\
P 14-24 & $-2.898 E-04$ & $6.854 E-03$ & 8.25 \\
P 15-25 & $7.246 E-04$ & $5.509 E-03$ & 6.63 \\
P 16-26 & $1.449 E-03$ & $1.204 E-02$ & 1.44 \\
P 41-31 & $0.520 E-03$ & $2.454 E-02$ & 2.95 \\
P 42-32 & $-1.449 E-04$ & $1.204 E-03$ & 1.49 \\
P 43-33 & $-2.898 E-04$ & $4.842 E-03$ & 5.82 \\
P 44-34 & $-8.695 E-04$ & $4.771 E-03$ & 5.74 \\
P 45-35 & 0.000 & $6.860 E-03$ & 8.25 \\
P 46-36 & $-2.898 E-03$ & $1.690 E-02$ & 2.03 \\
\hline \multicolumn{4}{|c|}{} \\
\hline TABLA 4.- CORRELACIÓN Y PRUEBA DE \\
MUESTRAS RELACIONADAS EN NIÑOS \\
\hline PARES & CORRELACIÓN & P \\
\hline P 11-21 & 0.987 & 0.894 \\
P 12-22 & 0.994 & 0.299 \\
P13-23 & 0.981 & 0.810 \\
P14-24 & 0.985 & 0.348 \\
P15-25 & 0.809 & 0.032 \\
P16-26 & 0.975 & 0.320 \\
P41-31 & 0.997 & 0.417 \\
P42-32 & 0.994 & 0.207 \\
P43-33 & 0.994 & 0.259 \\
P44-34 & 0.986 & 0.657 \\
P45-35 & 0.982 & 0.222 & 0.135 \\
P46-36 & 0,800 & \\
\hline
\end{tabular}

ERROR
TIP. MEDIA
7.369E-04
$8.296 \mathrm{E}-04$
$4.742 \mathrm{E}-04$
$8.251 \mathrm{E}-04$
$6.632 \mathrm{E}-04$
$1.449 \mathrm{E}-03$
$2.954 \mathrm{E}-03$
$1.499 \mathrm{E}-04$
$5.829 \mathrm{E}-04$
$5.744 \mathrm{E}-04$
$8.258 \mathrm{E}-04$
$2.034 \mathrm{E}-03$

\begin{tabular}{|c|c|c|}
\hline $\begin{array}{c}\text { INTERVALO } \\
\text { INFERIOR }\end{array}$ & $\begin{array}{c}\text { INTERVALO } \\
\text { SUIPERIOR }\end{array}$ & "t" \\
\hline$-2.340 \mathrm{E}-03$ & $6.009 \mathrm{E}-04$ & -1.180 \\
$-2.669 \mathrm{E}-03$ & $6.409 \mathrm{E}-04$ & -1.223 \\
$-2.395 \mathrm{E}-03$ & $5.030 \mathrm{E}-04$ & -3.056 \\
$-1.936 \mathrm{E}-03$ & $1.357 \mathrm{E}-03$ & -0.351 \\
$-5.987 \mathrm{E}-04$ & $2.048 \mathrm{E}-03$ & 1.093 \\
$-1.442 \mathrm{E}-03$ & $4.341 \mathrm{E}-03$ & 1.000 \\
$-3.431 \mathrm{E}-04$ & $1.343 \mathrm{E}-04$ & -1.000 \\
$-4.341 \mathrm{E}-04$ & $1.443 \mathrm{E}-04$ & -1.000 \\
$-1.453 \mathrm{E}-03$ & $8.733 \mathrm{E}-04$ & -0.497 \\
$-2.015 \mathrm{E}-03$ & $2.765 \mathrm{E}-04$ & -1.514 \\
$-1.647 \mathrm{E}-03$ & $1.648 \mathrm{E}-03$ & 0.000 \\
$-6.958 \mathrm{E}-03$ & $1.161 \mathrm{E}-03$ & -1.425 \\
\hline
\end{tabular}

TABLA 4.- CORRELACIÓN Y PRUEBA DE MUESTRAS RELACIONADAS EN NINOS

\begin{tabular}{|c|c|c|}
\hline \multicolumn{3}{|c|}{ TABLA 5.- CORRELACIÓN Y PRUEBA DE } \\
\multicolumn{3}{|c|}{ MUESTRAS RELACIONADAS EN NIÑAS } \\
\hline PARES & CORRELACIÓN & $\boldsymbol{P}$ \\
\hline P 11-21 & 0.992 & 0.242 \\
P 12-22 & 0.989 & 0.226 \\
P13-23 & 0.969 & 0.003 \\
P14-24 & 0.875 & 0.726 \\
P15-25 & 0.948 & 0.278 \\
P16-26 & 0.888 & 0.321 \\
P41-31 & 0.989 & 0.311 \\
P42-32 & 0.999 & 0.321 \\
P43-33 & 0.963 & 0.621 \\
P44-34 & 0.905 & 0.135 \\
P45-35 & 0.914 & 1.000 \\
P46-36 & 0.948 & 0.159 \\
\hline
\end{tabular}




\begin{tabular}{|}
\begin{tabular}{|c|c|c|c|c|c|c|}
\hline \multicolumn{7}{|c|}{ TABLA 6.- COMPARACIÓN DE LOS TAMAÑOS M-D ENTRE DIENTES HOMÓLOGOS } \\
EN D. TEMPORAL EN MAX Y MAND EN NIÑOS \\
\hline PARES & MEDIA & DES. TIP. & ERROR TIP. & INFERIOR & SUPERIOR & "t» \\
\hline P 53-63 & $1.569 E-03$ & $1.272 \mathrm{E}-02$ & $1.260 \mathrm{E}-03$ & $-9.303 \mathrm{E}-04$ & $4.068 \mathrm{E}-03$ & 1.245 \\
P 54-64 & $-1.960 \mathrm{E}-04$ & $1.980 \mathrm{E}-03$ & $1.961 \mathrm{E}-04$ & $-5.850 \mathrm{E}-04$ & $1.929 \mathrm{E}-04$ & -1.000 \\
P 55-65 & $1.078 \mathrm{E}-03$ & $2.110 \mathrm{E}-02$ & $2.090 \mathrm{E}-03$ & $-3.066 \mathrm{E}-03$ & $5.224 \mathrm{E}-03$ & 0.516 \\
P 73-83 & $3.922 \mathrm{E}-04$ & $1.062 \mathrm{E}-02$ & $1.051 \mathrm{E}-03$ & $-1.693 \mathrm{E}-03$ & $2.477 \mathrm{E}-03$ & 0.373 \\
P 74-84 & $1.176 \mathrm{E}-03$ & $9.152 \mathrm{E}-03$ & $9.061 \mathrm{E}-04$ & $-6.210 \mathrm{E}-04$ & $2.974 \mathrm{E}-03$ & 1.298 \\
P 75-85 & $1.110 \mathrm{E}-16$ & $1.990 \mathrm{E}-03$ & $1.970 \mathrm{E}-04$ & $-3.908 \mathrm{E}-04$ & $3.909 \mathrm{E}-04$ & 0.000 \\
\hline
\end{tabular}
\end{tabular}

\begin{tabular}{|c|c|c|c|c|c|c|}
\hline \multicolumn{7}{|c|}{ TABLA 6.- COMPARACIÓN DE LOS TAMAÑOS M-D ENTRE DIENTES HOMÓLOGOS } \\
EN D. TEMPORAL EN MAX Y MAND EN NINAS \\
\hline PARES & MEDIA & DES. TIP. & ERROR TIP. & INFERIOR & SUPERIOR & "t \\
\hline P 53-63 & $-4.637 \mathrm{E}-03$ & $3.632 \mathrm{E}-02$ & $4.373 \mathrm{E}-04$ & $-1.336 \mathrm{E}-02$ & $4.088 \mathrm{E}-03$ & -1.061 \\
P 54-64 & $-7.246 \mathrm{E}-04$ & $6.712 \mathrm{E}-03$ & $8.081 \mathrm{E}-04$ & $-2.337 \mathrm{E}-03$ & $8.878 \mathrm{E}-04$ & -0.897 \\
P 55-65 & $-2.898 \mathrm{E}-04$ & $3.824 \mathrm{E}-03$ & $4.603 \mathrm{E}-04$ & $-1.208 \mathrm{E}-03$ & $6.287 \mathrm{E}-04$ & -0.630 \\
P 73-83 & $2.339 \mathrm{E}-04$ & $8.186 \mathrm{E}-03$ & $6.260 \mathrm{E}-04$ & $-1.001 \mathrm{E}-03$ & $1.470 \mathrm{E}-03$ & 0.374 \\
P 74-84 & $1.449 \mathrm{E}-04$ & $1.204 \mathrm{E}-03$ & $1.449 \mathrm{E}-04$ & $-1.442 \mathrm{E}-04$ & $4.341 \mathrm{E}-04$ & 1.000 \\
P 75-85 & 0.000 & $7.859 \mathrm{E}-03$ & $9.461 \mathrm{E}-04$ & $-1.888 \mathrm{E}-03$ & $1.888 \mathrm{E}-03$ & 0.000 \\
\hline
\end{tabular}

\begin{tabular}{|c|c|c|c|c|c|c|}
\hline \multicolumn{7}{|c|}{ TABLA 6.- COMPARACIÓN DE LOS TAMAÑOS M-D ENTRE DIENTES HOMÓLOGOS } \\
EN D. TEMPORAL EN MAX Y MAND EN NINAS \\
\hline PARES & MEDIA & DES. TIP. & ERROR TIP. & INFERIOR & SUPERIOR & " $»$ \\
\hline P 53-63 & $-4.637 E-03$ & $3.632 E-02$ & $4.373 E-04$ & $-1.336 E-02$ & $4.088 E-03$ & -1.061 \\
P 54-64 & $-7.246 E-04$ & $6.712 E-03$ & $8.081 E-04$ & $-2.337 E-03$ & $8.878 E-04$ & -0.897 \\
P 55-65 & $-2.898 E-04$ & $3.824 E-03$ & $4.603 E-04$ & $-1.208 E-03$ & $6.287 E-04$ & -0.630 \\
P 73-83 & $2.339 E-04$ & $8.186 E-03$ & $6.260 E-04$ & $-1.001 E-03$ & $1.470 E-03$ & 0.374 \\
P 74-84 & $1.449 E-04$ & $1.204 E-03$ & $1.449 E-04$ & $-1.442 E-04$ & $4.341 E-04$ & 1.000 \\
P 75-85 & 0.000 & $7.859 E-03$ & $9.461 E-04$ & $-1.888 E-03$ & $1.888 E-03$ & 0.000 \\
\hline
\end{tabular}

\section{TABLA 8.- CORRELACIONES Y PRUEBA DE MUESTRAS RELACIONADAS}

\begin{tabular}{|l|c|c|c|c|}
\hline PARES & $\begin{array}{c}\text { CORRE- } \\
\text { LACIÓN } \\
\text { NIÑOS }\end{array}$ & $\begin{array}{c}\text { CORRE- } \\
\text { LACIÓN } \\
\text { NINAAS }\end{array}$ & $\begin{array}{c}\text { SIG. } \\
\text { (Bilateral) } \\
\text { P. NIÑOS }\end{array}$ & $\begin{array}{c}\text { SIG. } \\
\text { (Bilateral) } \\
\text { P. NINAAS }\end{array}$ \\
\hline P 53-63 & 0.919 & 0.973 & 0.216 & 0.293 \\
P 54-64 & 0.997 & 0.928 & 0.320 & 0.293 \\
P 55-65 & 0.961 & 0.993 & 0.607 & 0.373 \\
P 73-83 & 0.954 & 0.948 & 0.710 & 0.709 \\
P 74-84 & 0.937 & 0.999 & 0.197 & 0.321 \\
P 75-85 & 0.997 & 0.976 & 1.000 & 1.000 \\
\hline
\end{tabular}

todos los dientes, excepto para el $1^{\circ}$ molar inferior y $2^{\circ}$ premolar superior que la correlación es de 0,8. La correlación en niñas también es altísima encontramos una correlación próxima a 0,99 en incisivos centrales y laterales, en caninos superiores, en incisivos centrales y laterales y caninos inferiores y próxima a 0,9 en $1^{\circ}$ premolar y $2^{\circ}$ premolar superior e inferior y $1^{\circ}$ molar superior e inferior (Tabla 4, Tabla 5).

Al comparar el tamaño mesiodistal de los dientes temporales en el lado derecho e izquierdo, observamos que no existen diferencias significativas al aplicar la " $t$ " de Student apareada, ni en los niños ni en las niñas (Tabla 6, Tabla 7).

Existe una gran correlación entre dientes homólogos que va desde 0,919 para caninos superiores a 0,997 en $2^{\circ}$ molares inferiores en niños, y de 0,928 para el $1^{\circ}$ molar superior a 0,999 para el primer molar inferior (Tabla 8). 


\section{DISCUSIÓN}

\section{$1^{\circ}$. CONSIDERACIONES SOBRE LA TÉCNICA UTILIZADA.}

En los estudios biométricos de la dentición humana la elección tanto de la metodología como de las características de la muestra a utilizar depende del propósito específico de la investigación. En cuanto al método de la medición, hemos realizado mediciones directamente en boca basándonos en que no existen diferencias significativas entre las mediciones en boca y en los modelos. Hemos observado que con las diferentes técnicas para la confección de modelos se va incrementando el error, lo que se debe a la expansión posterior de la escayola $(15,16)$. Según Lavelle (17), las mediciones sobre modelos son un 2 $3 \%$ superiores que las realizadas sobre dientes naturales. Coleman (16) opina que se produce un error considerable por el uso de alginatos, yesos o jabones. También Moorrees (14) opina que los dientes tienden a ser ligeramente mayores que los originales, por la expansión inicial del alginato y la expansión posterior de la escayola, aunque la causa también puede deberse al desgaste de los puntos odontométricos, por sucesivas manipulaciones y al embellecimiento de los modelos. Plasencia (18) afirma que la única razón por la que un ortodoncista prescindiría de los modelos podría ser, que la propia boca del paciente es el más perfecto de los modelos.

\section{2․ COMPARACIÓN DEL DIÁMETRO MESIODISTAL ENTRE DIENTES HOMOLOGOS EN DENTICIÓN PERMANENTE}

Nosotros no hemos encontrado diferencias significativas entre los tamaños mesiodistales del lado derecho e izquierdo tanto en niños como en niñas, aunque las niñas presentan una ligera diferencia a nivel del 13 y 23 de $p=0,04$. Hemos obtenido unos niveles de simetría altísimos.

Coincidimos con numerosos estudios, en que no existe diferencias significativas entre dientes homólogos ( Moorrees y col 19, Sanin y Savara 20, Moyers y col 21, Margetts y Brown 22, Lysell y col 23, Axellsson y col 24, y Ostos, Travesí y González 25, entre otros).
Eduardo y García Godoy (26), encontraron que el único diente que presentaba diferencias significativas con su antímero fue el incisivo lateral superior, siendo más grande el del lado derecho.

Lysell y Murberg (23) obtuvieron unos coeficientes de correlación que varían entre 0,75 y 0,93 en la arcada superior y 0,78 y 0,90 en la arcada inferior. Ostos M.J. (25) en el maxilar superior obtiene un coeficiente que oscila entre 0,81-0,91 en niños y 0,79-0,91 en niñas, y en el maxilar inferior el rango osciló entre $0,84-0,88$ en niños y $0,77-0,89$ en niñas. Nosotros hemos obtenido unos niveles de correlación altísimos, al analizar la correlación entre dientes homólogos, observamos en niños una correlación de 0,99 para casi todos los dientes, excepto para el $1^{\circ}$ molar inferior y $2^{\circ}$ premolar superior que la correlación es de 0,8 . La correlación en niñas también es altísima encontramos una correlación próxima a 0,99 en incisivos centrales y laterales, en caninos superiores, en incisivos centrales y laterales y caninos inferiores y próxima a 0,9 en $1^{\circ}$ premolar y $2^{\circ}$ premolar superior e inferior y $1^{\circ}$ molar superior e inferior.

Para Kieser, Croeneveld y Preston (27), los indios Lengua mostraban mayor grado de asimetría derecha-izquierda que los Caucásicos. Para estos autores, el alto grado de desnutrición y enfermedad en esta población rural de Paraguay, sería el factor ambiental causante de la asimetría. En contraste la población Caucásica con dieta rica en proteinas y sin enfermedad estaría libre de estas alteraciones.

Otros autores como Bishara y col (28), encontraron diferencias significativas en 4 de las 12 parejas de dientes en población mejicana y en el $20 \%$ de las parejas de piezas en población norteamericana. Potter y col (93 tesis) en la población tagala observaron que una de cada cuatro piezas presentaba diferencias.

Keene determinó en negros norteamericanos que 11 de las 14 parejas posibles de dientes mostraban diferencias aunque estas nunca excedieron de $0,1 \mathrm{~mm}$. Macko y col evidenciaron igualmente en negros de Norteamérica diferencias derecha e izquierda en más del $80 \%$ de los casos, aunque estas eran menores de $0,5 \mathrm{~mm}$. 
Barret y col (29) encuentra diferencias entre los valores derechos e izquierdos dentro de una misma arcada ocasionalmente, especialmente en los terceros molares y en incisivo lateral superior, sin embargo las diferencias entre los valores promedios derechos y sus correspondientes izquierdos no son estadísticamente significativas.

Gran y col (30) indicaron que los dientes del lado izquierdo exceden a los del derecho en 16 de las 28 parejas, aunque sin significación estadística.

En 1997 Olayinka D, Otuyemi y Joe Noar H (31), realizaron el estudio en población nigeriana y británica, no se observaron diferencias significativas entre dientes homólogos $(\mathrm{p}<0,05)$, tampoco Keith en 1997 encontró diferencias, aunque encontraron asimetría en el $1^{\circ}$ molar y $2^{\circ}$ molar, pero dicha asimetría era pequeña. Los dientes posteriores eran menos variables que los dientes anteriores en ambas denticiones en los niños chinos, pero los dientes anteriores de cada clase morfológica eran en general menos variables que los dientes posteriores.

Los estudios efectuados en la raza caucásica indican que sus dientes son más estables que los de otras poblaciones donde existen con mayor frecuencia diferencias de tamaño entre dientes homólogos dentro de la misma arcada.

La aparición en la dentición de asimetrías casuales es atribuible a disturbios producidos por factores ambientales durante el desarrollo de la dentición. En el hombre, estudios en gemelos han demostrado que la asimetría en el tamaño dental por si misma no es hereditaria generalmente (32).

\section{3․ COMPARACIÓN DEL DIÁMETRO MESIODISTAL ENTRE DIENTES HOMÓLOGOS EN DENTICIÓN TEMPORAL.}

La asimetría del tamaño dentario resulta de interés para el odontopediatra y ortodoncista cuando se evalúan casos y planes de tratamiento.

Hemos encontrado diferencias mínimas entre las medias de los diámetros derechos-izquierdos y hemos encontrado unos niveles de simetría altísimos.
Al analizar la correlación entre dientes homólogos, vemos que nuestra correlación oscila entre 0,89 para incisivos centrales superiores y 1 en primeros molares permanentes superiores, incisivo central, caninos, primer molar temporal y primer molar permanente inferior.

Coincidimos con otros estudios en que no existen diferencias significativas entre los diámetros de los dientes del lado derecho y los del izquierdo $(23,33,24,22,34,21,25,14)$.

Algunos autores si han observado diferencias significativas entre dientes homólogos, como Gran (30), quien observó asimetría bilateral entre dientes homólogos, un diente de un lado puede ser mayor que su contralateral en 0,1-0,4 mm e incluso más, pero no se presenta una asimetría entre ambas hemiarcadas cuando se considera en conjunto, lo que, en su opinión, se debería a la existencia de un control genético que compensaría la diferencia.

\section{CONCLUSIONES}

1. No encontramos diferencias significativas entre las mediciones realizadas en la boca y en los modelos.

2. Hemos obtenido unos niveles de simetría muy elevados tanto en dentición temporal como permanente.

\section{BIBLIOGRAFÍA}

1. Keith K, Yuen W, Lisa L, So Y, Endarra L, Tang K. Mesiodistal crown diameters of the primary and permanent teeth in Southern Chinese, a longitudinal study. European Journal of Orthodontics 1997; 19: 721-31.

2. Steigman S, Harari D, Kuraita-Landman. Relationship between mesiodistal crown diameter of posterior deciduous and succedaneous teeth in Israeli Children. Eur. J. Orthod 1982; 4: 113-22. 
3. Santoro M, Ayoub ME, Pardi VA., Cangialosi TJ. Mesiodistal crown dimensions and tooth size discrepancy of the permanent dentition of Dominican Americans. Angle Ortho 2000; 70 (4): 303-7.

4. Kabban M, Fearne J, Jovanovski V, Zou L. Tooth size and morphology in twins. Int. J Paediatr Dent 2001; 11(5): 333-9.

5. Harila-Kaera V, Heikkinen t, Alvesalo L, Osborne $\mathrm{RH}$. Permanent tooth crown dimensions in prematurely born children. Early Hum Dev 2001; 62 (2): 131-47.

6. Haria S, Noar JH, Sanders R. An investigation of the dentition of parents of children with cleft lip and palate. Cleft Palate Craniofac J 2000; 37 (4): 395405.

7. Melo L, Ono Y, Takagi Y. Indicators of mandibular dental crowding in the mixed dentition. Pediatr Dent 2001; 23(2): 118-122.

8. Ngan P, Alkire RG., Fields H. Management of space problems in the primary and mixed dentitions. J. Am. Dent. Assoc 1999; 130 (9): 1330-9.

9. Seow WK, Wan A. A controlled study of the morphometric changes in the primary dentition of pre-term, very-low-birthweight children. J.Dent.Res 2000; 79(1): 63-69.

10. Liversidge HM, Molleson TI. Deciduous tooth size and morphogenetic fields in children from Christ Church, Spitalfieds. Arch Oral Biol 1999; 44 (1):7-13.

11. Bailit HL. The size and morphology of the Nasion dentition. Am.J. Phys.Anthrop 1968; 28: 271-88.

12. Black TK. Sexual dimorphism in the tooth-crwon diameters of the deciduous teeth. Am. J. Phys. Anthrop 1978; 48: 77-82.

13. Lundstrom A. Tooth size and occlusion in Twins. Br Dent J 1949; 87: 297-300.

14. Moorrees CFA, Reed RB. Correlations among crown diameters of human teeth. Arch Oral Biol 1964; 9: 685-97.
15. Hunter WS., Priest WR. Errors and discrepancies in measurement of tooth size. J Dent Res 1966;39: 405-13.

16. Coleman D. Mesiodistal crown dimensions of permanent teeth of Black Americans. ASDCJ Dent Child 1979; 46: 314-8.

17. Lavelle CLB. Metric analysis of primate tooth form. En: Butler, Joysey, editors. Development function and evolution teeth. Londres: Academic Press, 1978; 229-47.

18. Plasencia E. and Canut J.A. Los análisis odontométricos revisados. Rev. Esp. Ortod 1989; 19: 165-79.

19. Moorrees CFA and Reed RB. Biometrics of crowding and spacing of the teeth in the mandible. Am M Phys Antrop 1954; 12: 77-88.

20. Sanin C and Savara BS. An analysis of permanent mesiodistal crown size. Am J Orthod 1971; 59: 488-500.

21. Moyers RE. Handbook of Orthodontics. Yearbook Medical Publisher Inc. Chicago 1988.

22. Brown T, Margetts B, Towsend GC. Comparison of mesiodistal crown diameters of deciduous and permanent teeth in Australina Aboriginals. Aust Dent J 1980; 25: 28-33.

23. Lysell $L$ and Myrberg M. Mesiodistal tooth size in the deciduous and permanent dentitions. Eur $\mathrm{J}$ Orthod 1982; 4: 113-22.

24. Axelsson G, Kirveskari P. Crown size of permanent teeth in Icelanders. Acta Odontol Scand 1983; 41: 181-6.

25. Ostos MJ, Travesi J, Gonzalez R. Análisis de los diámetros mesiodistales dentarios en dentición permanente. Ortod Española 1983; 40: 233-43.

26. Towsend GC, García Godoy F. Fluctuating asymmetry in the deciduous dentition of Dominican Mulato children. Arch Oral Biol 1984; 29: 483-6. 